\title{
Role of Cardiac Magnetic Resonance Imaging in the Assessment of Myocardial Viability in Patients with Coronary Artery Disease
}

\author{
AALAA Y. MOHAMMED, M.Sc.; OMAR A. HASSANIEN, M.D.; AHMED S. IBRAHIM, M.D. and \\ NAGLAA L. DABEES, M.D. \\ The Department of Diagnostic Radiology and Medical Imaging, Faculty of Medicine, Tanta University, Tanta, Egypt
}

\begin{abstract}
Background: The principal goal of the assessment of myocardial viability is to identify patients whose symptoms, exercise capacity, and long-term prognosis may improve after revascularization. Contrast enhanced cardiac MRI has developed as a promising imaging modality for the assessment of myocardial viability which provides direct imaging of necrotic tissue with high contrast and high spatial resolution. Aim of the work was to assess role of cardiac MRI as a non-invasive tool in the evaluation of myocardial viability in patients with coronary artery disease.
\end{abstract}

Aim of Study: To assess the role of cardiac MRI as a noninvasive tool in the evaluation of myocardial viability in patients with coronary artery disease.

Material and Methods: The study included 25 patients diagnosed as having Coronary Artery Disease (CAD), (age range, 28-68 years \pm 11.29 ). All selected patients were subjected to cardiac MRI examination using 1.5-T MR system for studying myocardial function and viability. Comparison was made between echocardiography and cardiac MRI regarding segmental wall motion using the 17 -segment model of the American Heart Association for assessment of myocardial function. Delayed-enhancement MRI was performed to determine myocardial viability and scarring while black blood T2W sequence with fat suppression was done to detect cases with acute infarction. Microvascular occlusion was diagnosed using early Gadolinium enhancement sequence.

Results: Good agreement between cardiac MRI and echocardiography regarding segmental wall motion with $p$ value of 0.065 . Sensitivity and specificity of cardiac MRI for detecting SWMA were $93.6 \%$, and $44.4 \%$ respectively. Myocardial viability was evaluated by cardiac MRI with the use of end-diastolic wall thickness, imaging of early and late enhancement after contrast administration. Late gadolinium enhancement allowed assessing state of myocardium and visualizing myocardial scar with its quantification. Two segments showed $1-25 \%$ transmural extent of myocardial wall infarction, while 120 segments showed $71-100 \%$ extent and 261 segments showed no enhancing scar.

Correspondence to: Dr. Aalaa Y. Mohammed, The Department of Diagnostic Radiology and Medical Imaging, Faculty of Medicine, Tanta University, Tanta, Egypt
Conclusion: Cardiac MR imaging is an effective and reliable method for the evaluation of myocardial function as compared to echocardiography. Also it is effective and reliable method for the evaluation of myocardial viability being able to assess both the infarct size and trans-murality and accordingly helps to predict functional recovery after revascularization.

Key Words: Ischemic Heart Disease (IHD) - Segmental Wall Motion Abnormality (SWMA) - Cardiac Magnetic Resonance $(C M R)$ - Myocardial viability - Late gadolinium enhancement.

\section{Introduction}

CORONARY Artery Disease (CAD) is a common morbidity among the aging population and has a significant mortality. The treatment strategies are frequently between medical treatment and revascularization however, not every patient with CAD can benefit from coronary revascularization so, assessment of viable myocardium has an important prognostic implication. Patients with a substantial amount of dysfunctional but viable myocardium are likely to benefit from coronary revascularization and may show improvements in regional and global contractile function, symptoms, exercise capacity, and long-term prognosis [1-3].

Imaging modalities such as Positron Emission Tomography (PET) in combination with perfusion imaging or Single Photon Emission Computed Tomography (SPECT) have been used previously for the detection of myocardial viability however, these techniques are relatively time-consuming and expose the patient to radiation [4].

During the last two decades, Contrast Enhanced cardiac MRI (CE-MRI) has developed as a promising imaging modality for the assessment of myocardial viability. It provides direct imaging of necrotic tissue with high contrast and high spatial resolution. In patients with $\mathrm{CAD}$, the transmural 
extent of the scar tissue which can be determined by cardiac MRI, predicts functional recovery after myocardial revascularization $[\mathbf{5 , 6 ]}$.

Magnetic resonance imaging is able to assess the myocardial viability through a series of different techniques which can assess metabolic, functional, and morphological alterations and tissue characteristics, in addition to evaluating cellular viability. The technique most widely used and with the greatest potential for clinical use is delayed myocardial enhancement. This technique can identify in a simple and objective way areas of hyperintense signal in the myocardium after administration of the contrast agent, with excellent histologic correlation to characterize areas with infarction/ fibrosis [7].

\section{Patients and Methods}

\section{Study population:}

This prospective study was performed upon 25 patients already diagnosed as having coronary artery disease, (age range, 28-68 years; 22 males \& 3 females. Patients were referred to MRI unit at the Department of Radiology and Medical Imaging of our institute during the period from December 2015 to January 2017. Ethics Committee approval and informed written consent were obtained. Privacy and confidentiality of all patient data were guaranteed. All data provision were monitored and used for scientific purpose only.

All selected patients were subjected to cMRI examination using 1.5-T MR system for studying myocardial function and viability. The inclusion criterion was the presence of ischemic heart disease based on clinical symptoms and documented LV dysfunction by ECG, echocardiography in the cardiology department, history of percutaneous coronary intervention (11 patients) or previous attack of myocardial infarction (11 patients). $\mathrm{Pa}-$ tients enrolled in this study had to fulfill the following criteria; sinus heart rhythm, the ability to hold breath for accepted time (10-20 seconds) and normal serum creatinine.

The exclusion criteria included; haemo-dynamic instability, acute respiratory insufficiency, altered general status, the need for continuous monitoring incompatible with MRI confines, decompensated congestive heart failure (unable to lie flat during MRI), impaired renal excretory function (calculated Glomerular Filtration Rate less than $30 \mathrm{~mL} / \mathrm{min} / 1.73$ $\mathrm{m}^{2}$ ) unless on dialysis, history of nephrogenic systemic fibrosis, history of claustrophobia and the other general contraindication to MRI included:
Cardiac pacemaker, implantable defibrillator, cerebral aneurysm clip, neural stimulator (e.g. tensunit), any type of ear implant, ocular foreign body (e.g. metal shavings), any implanted device (e.g. insulin pump), metal shrapnel or bullet.

All patients of our study were subjected to full history taking, clinical examination: Including general examination and local examination by cardiologist, ECG monitoring, echocardiography and serum cardiac biomarkers estimation (e.g troponin) and cardiac MRI examination with the studying of myocardial function and viability.

\section{MR imaging protocol:}

This prospective study was performed in our institute using cardiac MRI examinations for 10 patients on closed MRI machine, 1.5-T magnet (Signa Excite HD; GE Healthcare, Milwaukee, USA) and 1.5-T MR system (Gyro scan Intera CV and Achiva; Philips Medical Systems, Best, the Netherlands) for 15 patients.

Localizer sequences in three orthogonal planes were taken followed by standard cardiac twochamber and four-chamber views as well as Short Axis (SA) cine images in Steady State Free Precession (SSFP). All images were acquired during single breath-holds (end expiratory of about 9$15 \mathrm{sec}$.) with ECG gating. Acquisition parameters for two-chamber and four-chamber views were: Repetition time msec/echo time msec, 4.2/1.8; flip angle, $55^{\circ}$; pixel size, $1.8-2.3 \mathrm{~mm}$; Field of View (FOV), 320 $\mathrm{mm}^{2}$; matrix, 192.

Short axis cine images acquisition parameters were: Repetition time msec/echo time msec, 4/1.6; flip angle, $55^{\circ}$; section thickness, $8 \mathrm{~mm}$; no gap; pixel size, 1.8-2.3mm; Field of View (FOV), 380 $\mathrm{mm}^{2}$; matrix, 280.

Contrast media was injected of about 0.2 $\mathrm{mmol} / \mathrm{kg}$ body weight with acquisition of real-time retrospective gated dynamic cine SA view in three slices in free breathing (at rest). Then, Late Gadolinium Enhancement (LGE) images were obtained $10 \mathrm{~min}$ after the injection of the contrast material using an Inversion Recovery (IR) sequence. Image acquisition was performed from the base to the apex in SA view and additionally in two chamber and four chamber view. Images were acquired during end-expiration breath hold.

Acquisition parameters of IR sequence are Repetition time msec/echo time msec, 7.4/3.4; section thickness, 8mm; no gap; Field of View (FOV), 380 $\mathrm{mm}^{2}$; matrix, 224; TI, variable for every patient. 


\section{Data processing for MRI:}

Functional image analysis:

Short axis CINE images were transferred to commercially available workstation for Postprocessing and image analysis. Images were transferred to the off-line workstation; advantage workstation (GE Healthcare Medical Systems, version 4.7) and also to another available work station; (extended work space "EWS"); Pride software (Philips Medical Systems).

The endocardial and epicardial borders of both ventricles were traced manually from the short axis images cine images during peak systole and end diastole giving global functional parameters (EF). The impact of IHD on regional function was assessed qualitatively using SA cine images (described as normal, hypokinetic, akinetic, or dyskinetic) using the 17-segment model.

\section{Morphological assessment:}

The end diastolic wall thickness was assessed to detect cases with reduced end diastolic wall thickness in chronic state. Also, the functional 2 chamber and 4 chamber cine images were assessed for other associated abnormalities such as valvular lesion or intra-cavitary thrombus.

The presence of edema to determine acute cases was assessed in the morphological short axis black blood T2WIs qualitatively as an area of high signal intensity than normal myocardium.

\section{Viability analysis:}

Visual assessment of early gadolinium enhancement images for the presence of any hypo-intense signal that denoted microvascular occlusion was also performed. Thereafter, visual assessment of late gadolinium enhancement images for the presence of scar tissue and its extent across the myocardial wall was performed using the identical 17segment model. The basal, mid-ventricular, and apical segments were evaluated on short-axis images, whereas the apical cap was evaluated in the two-chamber or four-chamber long-axis planes. Transmural extent of infarction was expressed as the following: $1-25 \%$ of left-ventricular wall thickness or $26-50 \%$ of $\mathrm{LV}$ wall thickness or $51-75 \%$ LV wall thickness and 76-100\% of LV wall thickness.

\section{Statistical analysis:}

- Statistical analysis was processed by using SPSS program, Version 16 of IL, USA.

- For quantitative data, the range, mean and standard deviation were calculated. For qualitative data, which describe a categorical set of data by frequency, percentage or proportion of each category, comparison between two groups and more was done using Chi-square test $(\chi 2)$ and Fisher Exact test. For comparison between percents testing of proportion was done (Z-test). Significance was adopted at $p<0.05$ for interpretation of results of tests of significance.

- For people who had the characteristic (segmental wall motion abnormality), the number of people who tested positive and the number of people who tested negative were recorded. The same was done for people who didn't have the characteristic. People with the characteristic and tested positive (enhanced scared myocardium on LGE in cMRI) are the True Positives (TP). People with the characteristic (SWMA) and tested negative (no enhanced scared myocardium on LGE in cMRI) are the False Negatives (FN). People without the characteristic (No SWMA) and tested positive are the False Positives (FP). People without the characteristic and tested negative are the True Negatives (TN).

- To calculate the sensitivity, TP was divided by $(\mathrm{TP}+\mathrm{FN})$.

- To calculate the specificity, TN was divided by $(\mathrm{FP}+\mathrm{TN})$.

- To calculate the Positive Predictive Value (PPV), TP was divided by (TP + FP).

- To calculate the Negative Predictive Value (NPV), TN was divided by $(\mathrm{TN}+\mathrm{FN})$.

- Accuracy, or efficiency, is the percentage of test results correctly identified by the test, i.e. (true positives+true negatives $) /$ total test results $=(\mathrm{TP}$ $+\mathrm{TN}) /(\mathrm{TP}+\mathrm{TN}+\mathrm{FP}+\mathrm{FN})$.

\section{Results}

Among the twenty five (25) patients included in our study, males represented $88 \%$ \& females represented $12 \%$. (22 males, 3 females), the age ranged between (28-68 years); with mean age: 48.68 years; standard deviation: \pm 11.29 .

According to the predisposing factors for developing coronary artery disease, hypertension was the most common finding as it was seen in 17 patients $(68 \%)$ followed by hypercholesterolemia which was present in 10 patients $(40 \%)$ and then diabetes mellitus which was present in 7 patients (28\%). 14 patients $(56 \%)$ were smokers, 6 patients (24\%) were drug addicts, 14 patients $(56 \%)$ had positive family history of coronary artery disease (Chart 1). 
Good agreement between Echocardiography and MRI for the estimation of Ejection Fraction (EF) was noted. In this study, the mean EF by cMRI was $43.92 \%$ and the mean EF by echocardiography was $39.87 \%$ with mean difference was $4.05 \%$.

As regard the detection of segmental wall motion abnormality, echocardiography showed that 149 segments were normo-kinetic while cMRI revealed that 140 segments were normo-kinetic. Also, echocardiography showed that 206 segments showed hypo-kinesia while cMRI showed that only 186 segments were hypo-kinetic. 67 segments were seen to be akinetic by echocardiography while 91 segments were seen to be akinetic by cMRI. Only 3 segments showed dyskinesia by echocardiography while, 8 segments showed dyskinesia by cMRI. So there was difference between cMRI and echocardiography as regard detection of segmental wall motion abnormality as described however it is insignificant with the $p$-value $=0.065$ (Table 1).

On comparing types of segmental wall abnormality detected by both echocardiography and cMRI, there was some difference in the type of SWMA as following (Table $2 \&$ Chart 2).

It was found that 149 segments appeared to be normo-kinetic by echocardiography. Only 77 segments $(51.7 \%)$ of them were normo-kinetic by both echocardiography and cMRI and 56 segments (37.6\%) appeared normo-kinetic by echo but they showed hypo-kinesia by cMRI. Also, 16 segments $(10.7 \%)$ appeared normo-kinetic by echo but they showed akinesia by cMRI.

Also, 206 segments appeared to be hypo-kinetic by echocardiography. Only 99 segments of them (48.1\%) were hypo-kinetic by both echocardiography and cMRI and 51 segments $(24.8 \%)$ appeared hypo-kinetic by echo but they were normo-kinetic by cMRI and 52 segments (25.2\% showed hypokinesia by echo but they were akinetic by cMRI. Moreover, 4 segments (1.9\%) were hypo-kinetic by echo but they were found to be dys-kinetic by cMRI.

By echocardiography, 67 segments appeared to be akinetic. Only 23 segments (34.3\%) of them were akinetic by both echocardiography and cMRI while 12 segments (17.9\%) appeared akinetic by echo but they were normo-kinetic by cMRI and 31 segments $(46.3 \%)$ showed akinesia by echo but they were hypo-kinetic by cMRI. Moreover, 1 segment $(1.5 \%)$ appeared akinetic by echo but it was found to be dys-kinetic by cMRI.
For the prediction of the contractile reserve in the studied 25 patients, assessment of Segmental Wall Motion Abnormality (SWMA) identified with cMRI associated with scar tissue as diagnosed by LGE was studied in the collectively three vascular territories (LAD, LCX \& RCA). A total number of 75 territories (in the studied 25 patients; three vascular territories in each patient) were studied. It was found that 58 territories of the 75 territories $(77.3 \%)$ showed abnormal regional function, whereas 40 of them (53.3\%) showed scar by LGE and 18 territories (24\%) showed no scar. Seventeen segments $(22.7 \%)$ showed no SWMA with only three of them (4\%) showed scar by cMRI (Table 3).

The same was done for echocardiography and it was found that 56 territories of 75 territories (74.6\%) showed abnormal regional function, whereas 36 of them (48\%) showed scar by LGE and 20 territories $(26.6 \%)$ showed no scar. Nineteen segments $(25.4 \%)$ showed no SWMA with only five of them $(6.7 \%)$ showed scar by cMRI.

Cardiac MRI provided the ability to differentiate cases with acute myocardial infarction from chronic cases by the use of morphological black blood T2 weighted imaging with fat suppression and it was found that 12 patients (48\%) showed abnormally high signal intensity in T2WI in the jeopardized myocardium denoting myocardial edema and hence acute myocardial infarction and 13 patients $(52 \%)$ had chronic ischemic heart disease.

With the use of LGE to detect myocardial scar tissue; 23 patients $(92 \%)$ showed abnormal high signal intensity involving some segments denoting myocardial infarction and 2 patients $(8 \%)$ showed no evidence of enhancing scar tissue denoting totally viable myocardium.

Regarding the extent of myocardial scar tissue, this study revealed that 2 segments showed $1-25 \%$ transmural extent of the myocardial wall infarction, 34 segments showed 26-50\% transmural extent of infarction, 8 segments showed $51-70 \%$ transmural extent of infarction and 120 segments showed $71-100 \%$ transmural extent of infarction while 261 segments showed no enhancing scar tissue (Table 4).

By LGE; segment 13 (apical-anterior segment) which is a LAD territory was found to be the most commonly affected segment (scarred) (noted in 15 cases; $60 \%$ ).

The left anterior descending artery (LAD) was found to be the most commonly affected coronary 
artery among the 23 patients showing scar tissue on LGE being involved in 18 patients (78.3\%).

Evidence of microvascular occlusion was found in 9 patients (39.1\%) among the 23 patients who revealed scar tissue on LGE denoting poorer prognosis (Table 5).

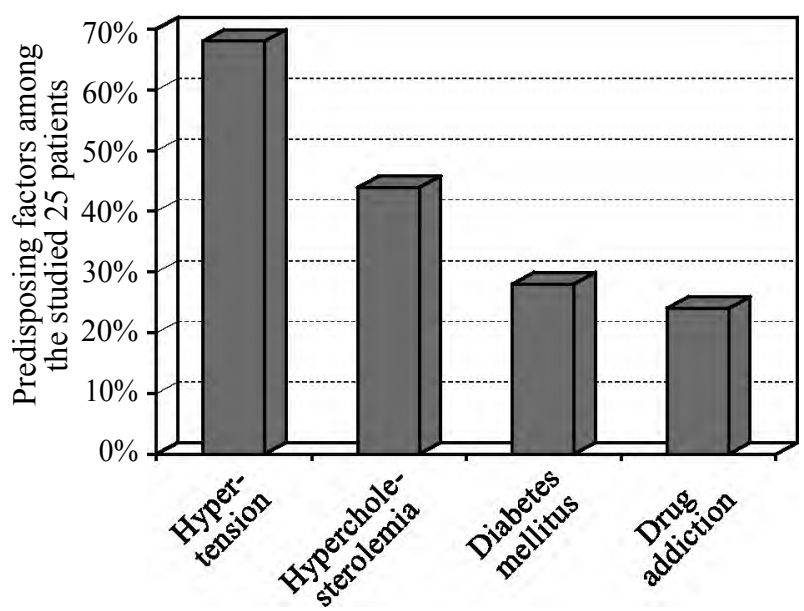

Chart (1): Important predisposing factors among the studied 25 patients having ischemic heart disease.

Table (1): Segmental wall motion abnormality diagnosed by Echocardiography (ECHO) versus cardiac Magnetic Resonance Imaging (cMRI) among the studied 25 patients having ischemic heart disease.

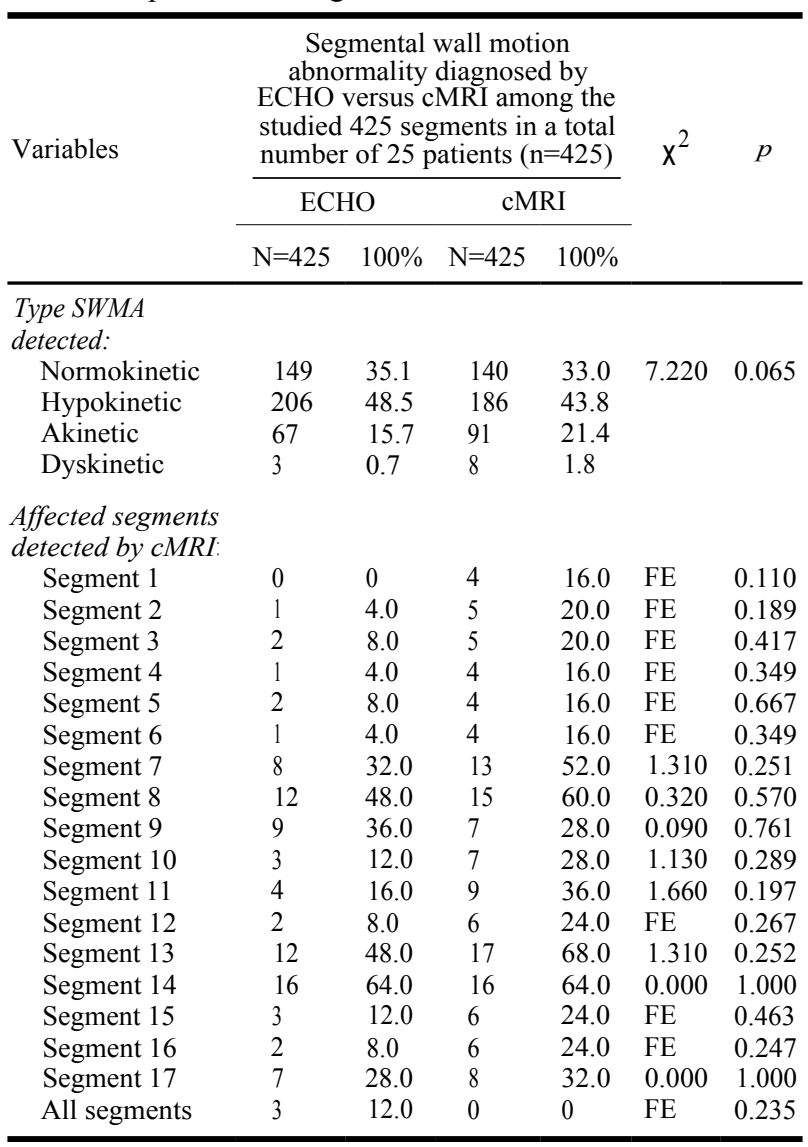

*: Significant $(p<0.05)$.

FE: Fisher Exact test.
Table (2): Per-segment agreement between echocardiography and MRI as regards detection of SWMA in the 425 segments examined in 25 patients.

\begin{tabular}{|c|c|c|c|c|c|}
\hline & \multicolumn{3}{|c|}{ Normal Hypokinesia Akinesia } & \multirow[t]{2}{*}{ Dyskinesia } & \multirow[t]{2}{*}{ Total } \\
\hline \multicolumn{4}{|l|}{ SWMA by } & & \\
\hline \multicolumn{6}{|l|}{ cMRI: } \\
\hline \multicolumn{6}{|l|}{ Normal: } \\
\hline Count & 77 & 51 & 12 & 0 & 140 \\
\hline$\%$ of Total & $51.7 \%$ & $24.8 \%$ & $17.9 \%$ & $0 \%$ & $33 \%$ \\
\hline \multicolumn{6}{|l|}{ Hypokinesia: } \\
\hline Count & 56 & 99 & 31 & 0 & 186 \\
\hline$\%$ of Total & $37.6 \%$ & $48.1 \%$ & $46.3 \%$ & $0 \%$ & $43.8 \%$ \\
\hline \multicolumn{6}{|l|}{ Akinesia: } \\
\hline Count & 16 & 52 & 23 & 0 & 91 \\
\hline$\%$ of Total & $10.7 \%$ & $25.2 \%$ & $34.3 \%$ & $0 \%$ & $21.4 \%$ \\
\hline \multicolumn{6}{|l|}{ Dyskinesia: } \\
\hline Count & 0 & 4 & 1 & 3 & 8 \\
\hline$\%$ of Total & $0 \%$ & $1.9 \%$ & $1.5 \%$ & $100.0 \%$ & $1.8 \%$ \\
\hline \multicolumn{6}{|l|}{ Total: } \\
\hline Count & 149 & 206 & 67 & 3 & 425 \\
\hline$\%$ of Total & $100.0 \%$ & $100.0 \%$ & $100.0 \%$ & $100.0 \%$ & $100.0 \%$ \\
\hline
\end{tabular}

Agreement between echocardiography and cMRI as regard detection of SWMA in the 425 segments

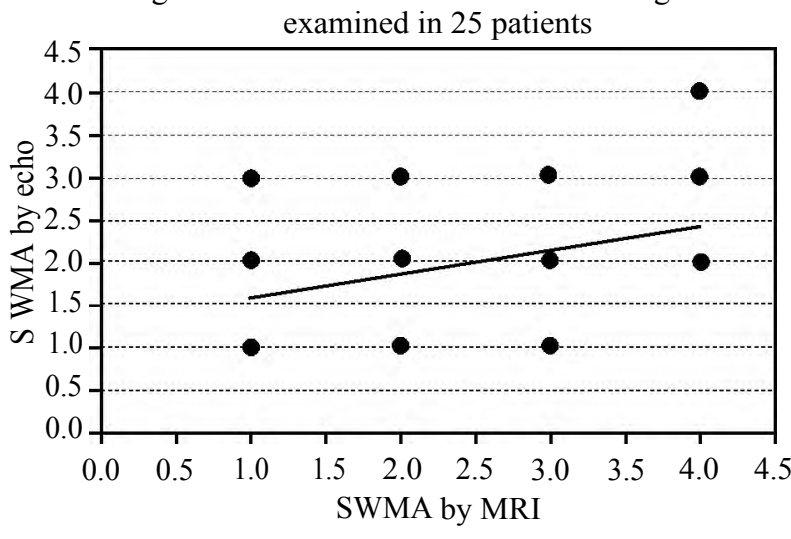

1: Normal. 2: Hypokinesia. 3: Akinesia 4: Dyskinesia

Chart (2): Demonstrating the agreement between echocardiography and cMRI as regard detection of different types of segmental wall motion abnormality.

Table (3): Diagnostic value of SWMA identified with cMRI for the prediction of myocardial scar as diagnosed by cMRI in the three vascular territories (LAD, LCX \& RCA)

\begin{tabular}{|c|c|c|c|}
\hline & \multicolumn{3}{|c|}{ Myocardial Scar by MRI } \\
\hline & Scar & No scar & Total \\
\hline \multicolumn{4}{|l|}{ SWMA detected by cMRI: } \\
\hline Motion & 40 & 18 & 58 \\
\hline Abnormality & $53.3 \%$ & $24 \%$ & $77.3 \%$ \\
\hline No motion & 3 & 14 & 17 \\
\hline Abnormality & $4 \%$ & $18.7 \%$ & $22.7 \%$ \\
\hline \multirow[t]{2}{*}{ Total } & 43 & 32 & 75 \\
\hline & $57.3 \%$ & $42.7 \%$ & $100 \%$ \\
\hline Sensitivity & $93.6 \%$ & & \\
\hline Specificity & $44.4 \%$ & & \\
\hline Positive Predictive Value (PPV) & $68.8 \%$ & & \\
\hline Negative Predictive Value (NPV) & $66.7 \%$ & & \\
\hline Accuracy & $72 \%$ & & \\
\hline
\end{tabular}


Table (4): Late Gadolinium Enhancement (LGE) diagnosed by cardiac Magnetic Resonance Imaging (cMRI) among the studied 425 left ventricular segments in the 25 patients having ischemic heart disease were included in this study.

\begin{tabular}{|c|c|c|}
\hline \multirow[t]{2}{*}{ Variable } & \multicolumn{2}{|c|}{$\begin{array}{l}\text { The studied patients with } \\
\text { ischemic heart disease }(n=25)\end{array}$} \\
\hline & $\mathrm{n}$ & $\%$ \\
\hline \multicolumn{3}{|l|}{$\begin{array}{l}\text { (A) Late gadolinium } \\
\text { enhancement (LGE): }\end{array}$} \\
\hline $\begin{array}{l}\text { - No scar tissue } \\
\text { (Totally viable myocardium) }\end{array}$ & 2 & 8.0 \\
\hline \multirow[t]{2}{*}{ - Evident enhancing scar tissue } & 23 & 92.0 \\
\hline & $\begin{array}{r}\text { Total numbe } \\
\text { left ventricu } \\
\text { the } 25 \text { patients in }\end{array}$ & $\begin{array}{l}\text { studied } 425 \\
\text { egments in } \\
\text { led in the study }\end{array}$ \\
\hline (B) Extension of the scar: & $\mathrm{n}=425$ & $100 \%$ \\
\hline • No enhancing scar tissue & 261 & $61.41 \%$ \\
\hline - $1-25 \%$ of myocardial wall & 2 & $0.47 \%$ \\
\hline - $25-50 \%$ of myocardial wall & 34 & $8.00 \%$ \\
\hline - $50-75 \%$ of myocardial wall & 8 & $1.88 \%$ \\
\hline - $75-100 \%$ of myocardial wall & 120 & $28.2 / 4 \%$ \\
\hline
\end{tabular}

Table (5): Microvascular Occlusion (MVO) diagnosed by cardiac Magnetic Resonance Imaging (cMRI) among the studied 23 patients showing scar tissue on LGE.

\begin{tabular}{llc}
\hline Variable & $\begin{array}{c}\text { The studied patients with } \\
\text { ischemic heart disease }(\mathrm{n}=23)\end{array}$ \\
\cline { 2 - 3 } & $\mathrm{n}$ & $\%$ \\
\hline Microvascular obstruction $(M V O)$ : & 14 & 60.9 \\
No & 9 & 39.1 \\
Present & & \\
Affected territory with MVO: & 8 & 34.7 \\
Left anterior descending artery (LAD) & 8.3 \\
Right coronary artery (RCA) & 1 & 4.3 \\
\hline
\end{tabular}

\section{Illustrated cases are shown:}

Case (1): Fig. (1), Case (2): Fig. (2), Case (3): Fig. (3), Case (4): Fig. (4).

Fig. (1) (A-G): 43-years old-male patient, hypertensive and heavy smoker, presented clinically with acute onset of dyspnea and chest tightness followed by syncopal attack. Echocardiography revealed hypo-kinesia of the apical and mid-anterior and antero-septal and antero-lateral segments with $\mathrm{EF}=44 \%$. Cardiac MRI revealed evidence of transmural myocardial scarring together with regional wall motion abnormality and moderate transmural myocardial edema following LAD and LCX territories associated with mildly decreased LV function denoting acute MI with low salvageable index as follows:

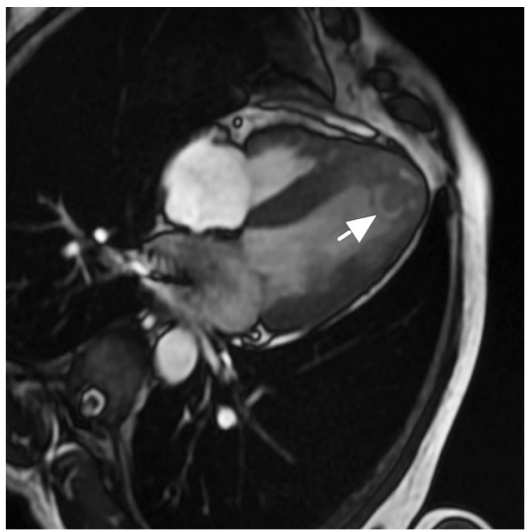

(A)

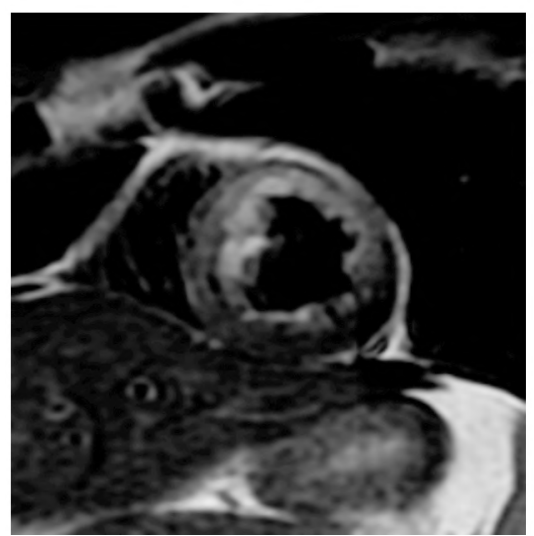

(D)

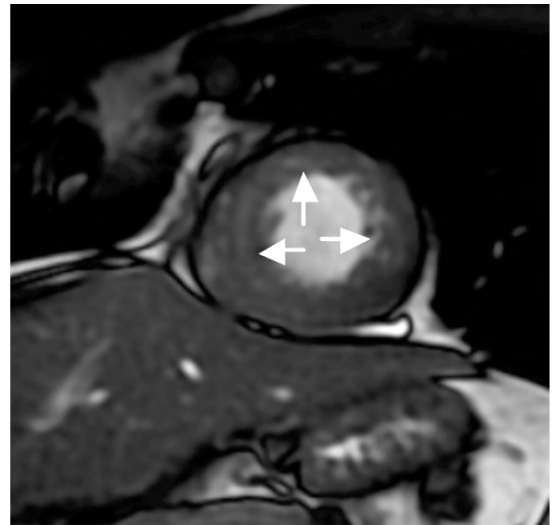

(B)

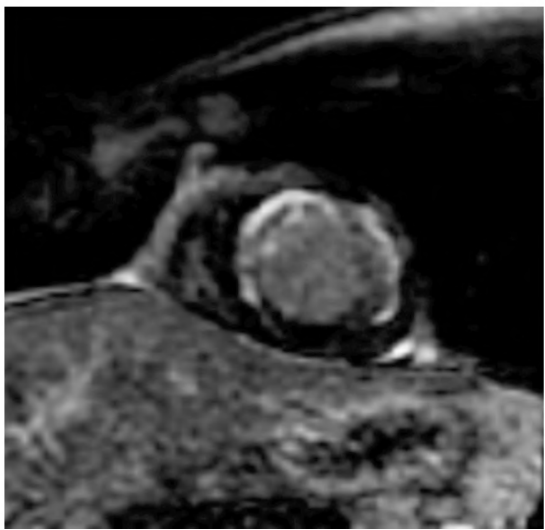

(E)

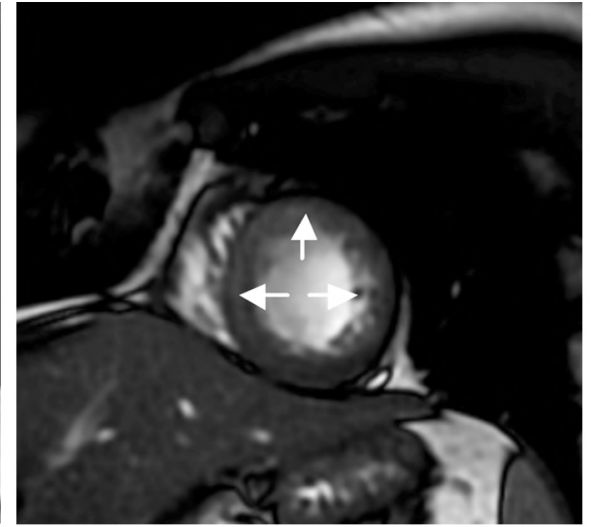

(C)

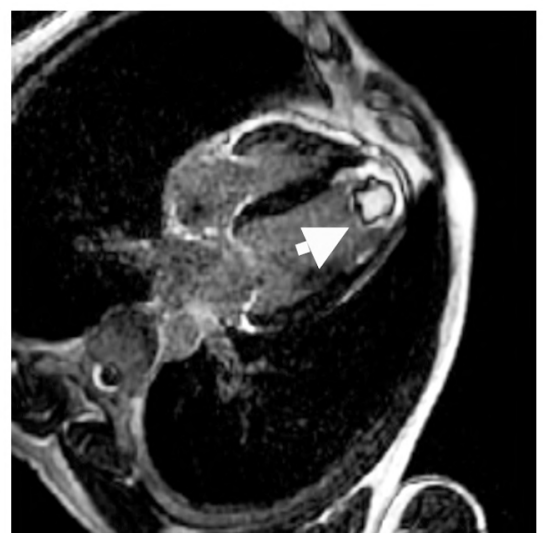

(F) 


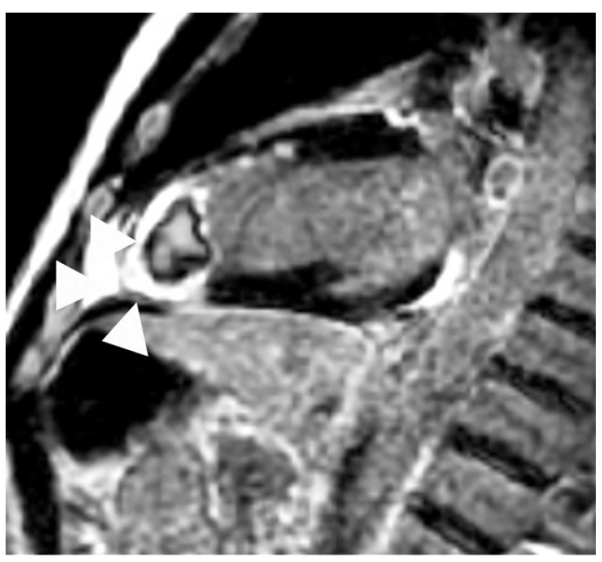

(F)
Fig. (1): (A): SSFP in 4 chamber view showing intra-luminal mass lesion (yellow arrow) seen within the left ventricle near the apex. (B,C): Cine MRI (gradient-echo sequence) in short axis view. (B) In peak systole and (C) In end diastole revealing akinesia of apical anterior, septal and lateral segments. Left ventricular ejection fraction was decreased $=48 \%$. (D): Short axis view, T2 weighted image showing transmural high signal intensity (white arrows) at apical-anterior, septal and lateral segments denoting transmural edema (areas at risk). Fig. (1E,F,G): Delayed enhancement (inversion recovery gradient-echo sequence), (E) In short axis view, (F) In 4 chamber view, and (G) in 2 chamber view revealed transmural infarction of the apex (yellow arrows in 1-D-4), Apical-anterior, septal, and lateral segments (Following LAD and LCX territories) associated with a LV apical thrombus (no enhancement within the mass confirmed its thrombotic nature).

Fig. (2A-F): 36-years old-male patient, heavy smoker, addict with positive family history of CAD. The patient was presented by recurrent attacks of typical chest pain, maximum 4 hours before admission. Echocardiography revealed hypo-kinesia of the mid-anterior, antero-septal, infero-septal segments as well as the apical-anterior and septal segments with $\mathrm{EF}=52 \%$. Cardiac MRI revealed ischemic heart disease, regional LAD hypokinesia, edematous changes following LAD territory denoting acute ischemia, small MVO with trans-mural infarction following LAD territory; overall bad reperfusion with low salvageable index.

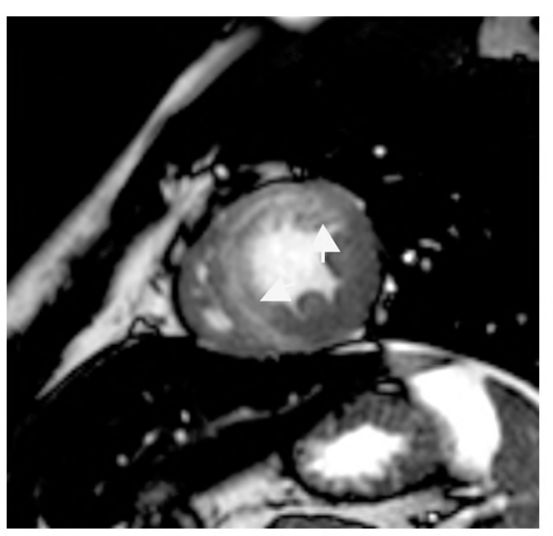

(A)

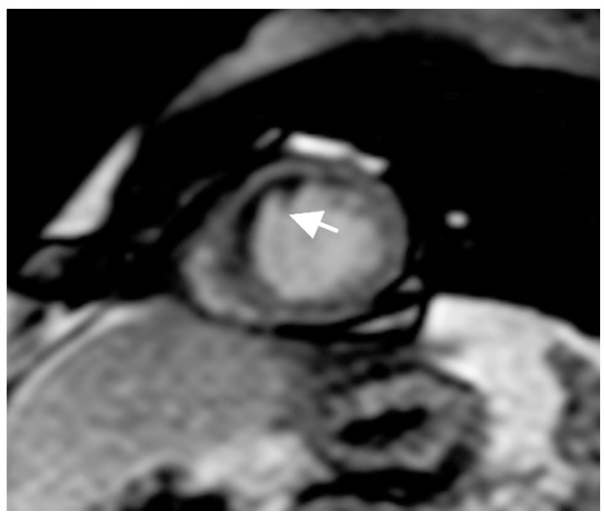

(D)

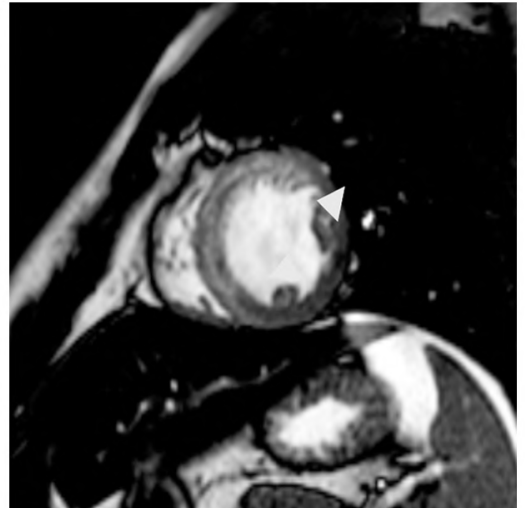

(B)

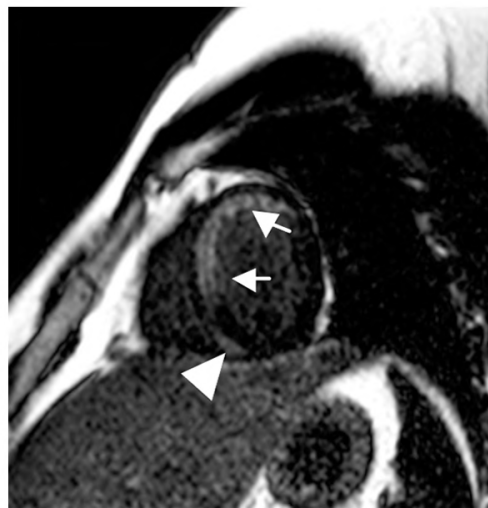

(E)

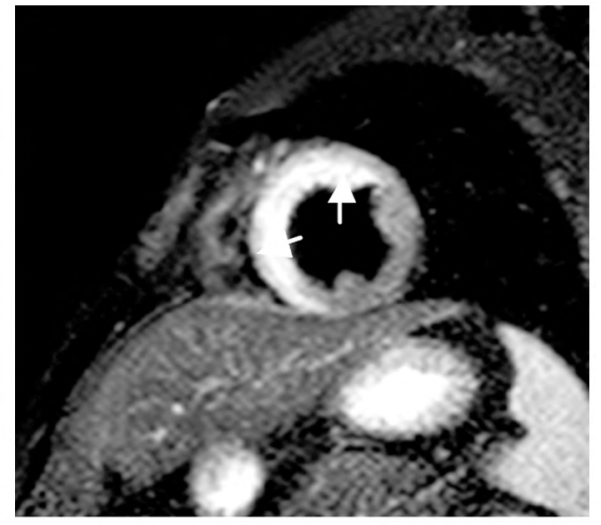

(C)

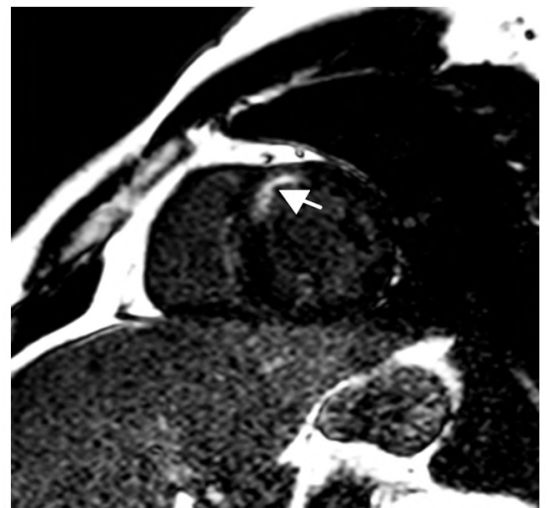

(F)

Fig. (2): (A,B): Cine MRI (gradient echo sequence) in short axis view. (A) In peak systole and (B) In end diastole revealing hypo-kinesia of apical anterior and septal segments. Left ventricular EF was measured $=61 \%$. Fig. (2C): Short axis view, T2 weighted imaging with fat suppression, showing trans-mural high signal intensity (white arrows) at apical-anterior and apicalseptal segments (areas at risk). Fig. (2D): Early post-gadolonium enhancement (T1 turbo spin-echo sequence), showing subendocardial hypo-intense zone of micro-vascular obstruction (yellow arrows) LAD territory. Fig. (2E,F): Delayed enhancement (inversion recovery gradient-echo sequence), in short axis views revealed apical, mid and basal anterior and antero-septal transmural infarction $(>75 \%)$ (yellow arrows) following LAD territory. 
Fig. (3A-G): 52-years old-male patient, diabetic, hypertensive with hyper-cholesterolemia. Status post-PCI after 5 days which revealed LAD, LCX and RCA significant stenosis with past-history of previous CABG 7 years ago. Echocardiography revealed Hypo-kinesia of them apex, apico-anteriro and apical-septal and lateral segments with moderately reduced $\mathrm{EF}=30 \%$. Cardiac MRI revealed ischemic heart disease with trans-mural infarction following LAD and LCX territory, associated with apical persistent micro-vascular occlusion, left ventricular dilatation and reduced systolic function $(\mathrm{EF}=32 \%)$, small subendocardial infarction is also noted following RCA territory and inferior papillary muscle scarring is also noted as follows:

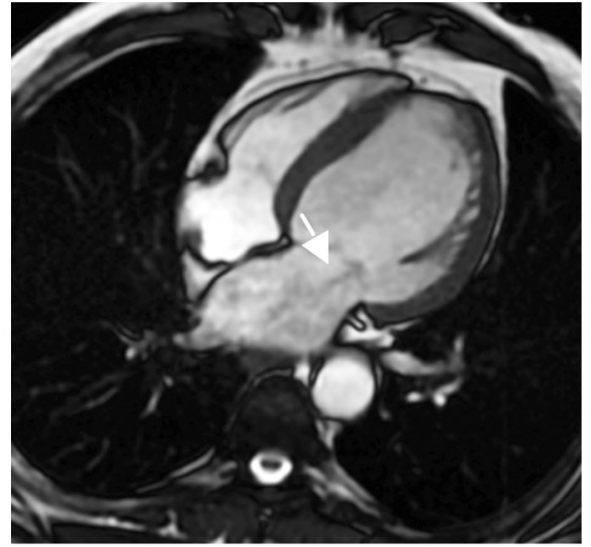

(A)

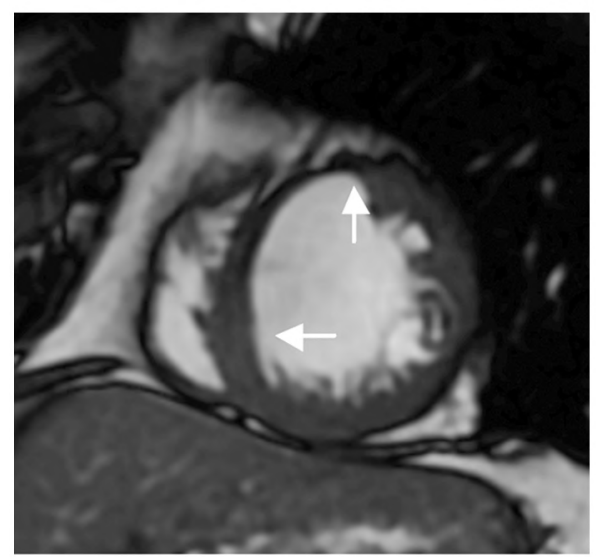

(C)

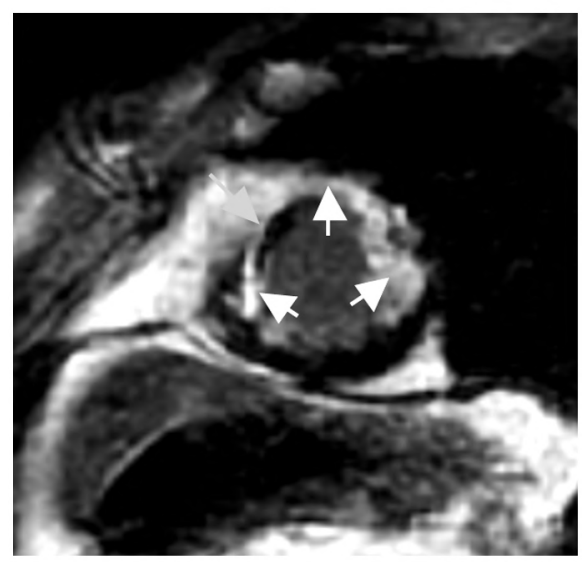

(E)

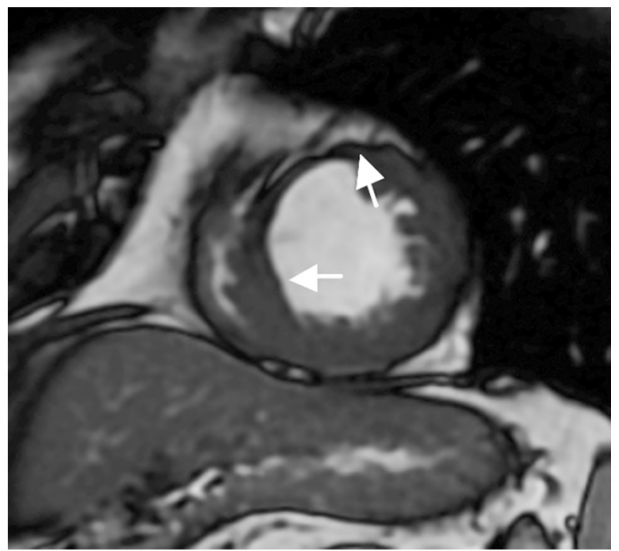

(B)

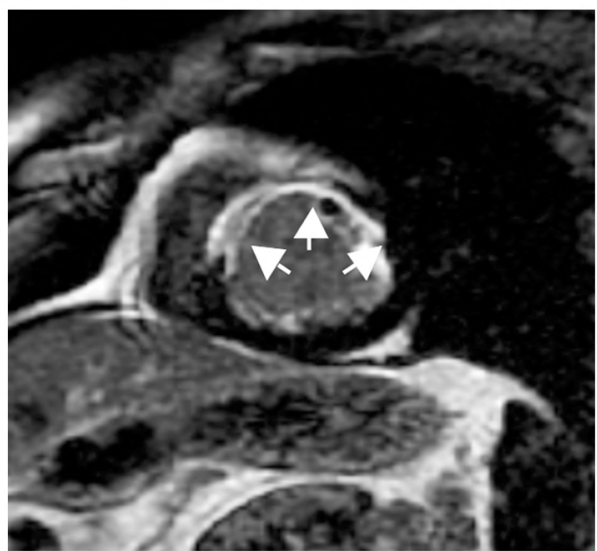

(D)

Fig. (3): (A): Cine MRI (gradient echo sequence) in 4 chamber view reveals dilatation of the four cardiac chambers. Evidence of mitral regurgitation is also noted (yellow arrow). (B,C): Cine MRI (gradient echo sequence) in short axis view. (B) In peak systole and (C) In end diastole revealing akinesia of the apical-anterior and apical-septal segments (yellow arrows) with thinning of the apicoantero-septal segment. Left ventricular EF was decreased $=32 \%$. Fig. (3D,E): Delayed enhancement (inversion recovery gradientecho sequence) (D,E) In short axis. Persistent micro-vascular occlusion is also noted appearing as sub-endocardial dark zone at the apical anterior segment (E, yellow arrow). 
Fig. (4A-F): 64-years old-male patient, diabetic and hypertensive, presented clinically with typical chest pain, exertional dyspnea and paroxysmal nocturnal dyspnea 6 days ago. Echocardiography revealed akinesia of the apical and mid-inferior, basal-anterior segments with hypo-kinesia of mid (anterior, septal and lateral) segments with impaired systolic function; $\mathrm{EF}=$ $38 \%$. Also, LA and LV dilatation was noted as well as mitral, tricuspid and aortic incompetence. Cardiac MRI revealed ischemic heart disease, edematous changes following LAD territory denoting acute ischemia. Regional LAD hypo-kinesia and RCA akinesia with no evidence of late gadolinium enhancement at the affected segments denoting dysfunctional but completely viable myocardium as follows:

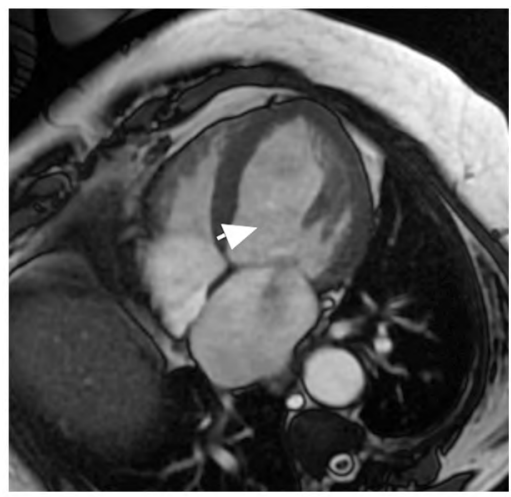

(A)

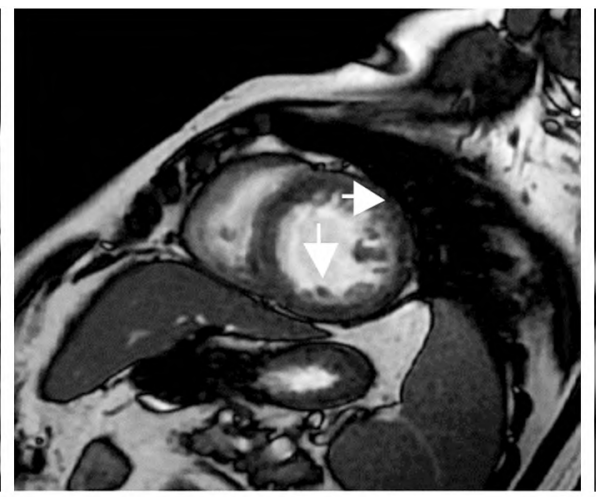

(B)

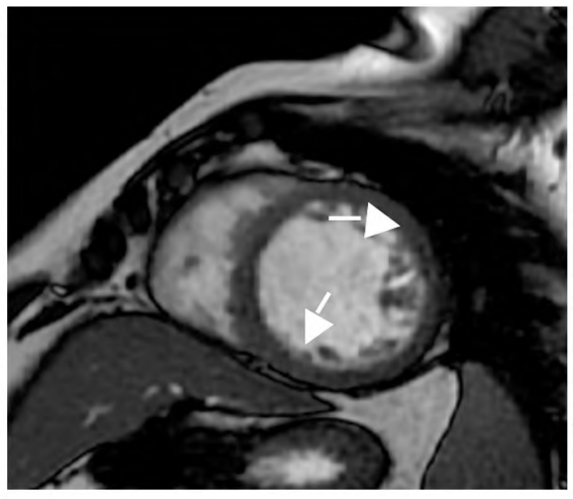

(C)
(D)

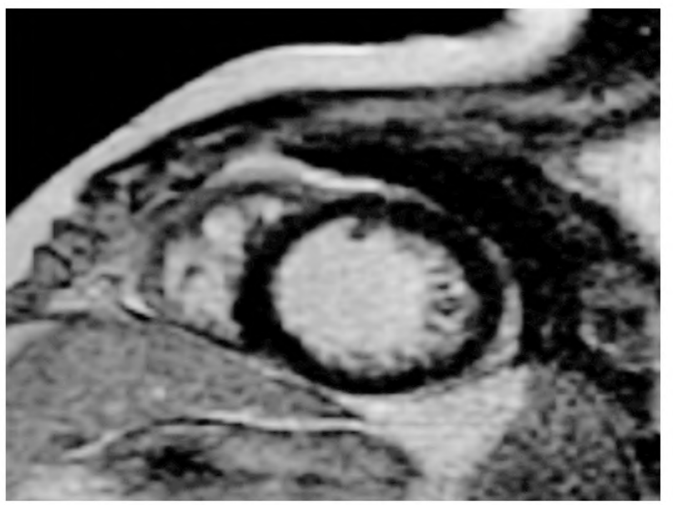

(F)

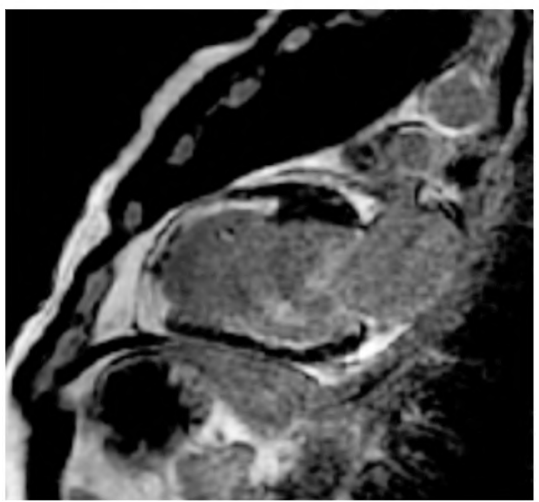

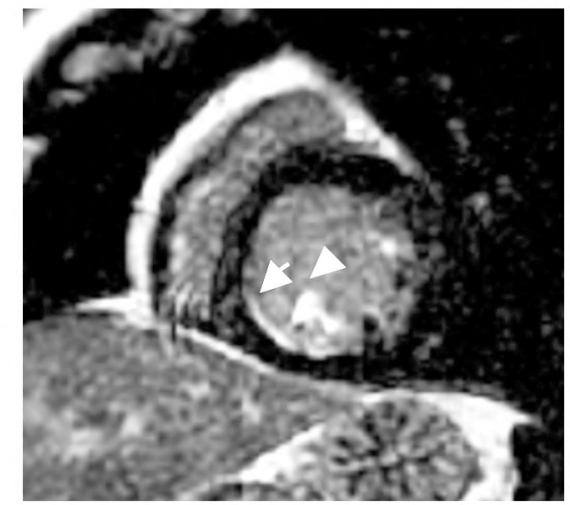

(E)

Fig. (4): (A): Cine MRI (gradient echo sequence) in 4 chamber view reveals dilatation of the four cardiac chambers with evidence of mitral regurgitation (white arrow). Fig. (4B,C): Cine MRI (gradient echo sequence) in short axis view, (B) In peak systole and (C) In end diastole revealing akinesia (white arrows) of the apical inferior and lateral and mid-inferior and infero-lateral segments (RCA territory) together with hypo-kinesia of apical and mid (anterior, septal and lateral) segments. Left ventricular EF was decreased=21\%. Fig. (4D,E,F): Delayed enhancement (inversion recovery gradient-echo sequence), (D) In short axis views (E) In 4 chamber view, and (F) In 2 chamber view showing no evidence of late enhancement at any segment.

\section{Discussion}

Coronary artery disease, accounts for about $2 / 3$ of cases of congestive heart failure and LV dysfunction which is often not the result of irreversible scar but rather caused by functional impairment of still viable-myocytes, with the opportunity for improved function if coronary blood flow is restored. Those patients benefit from revascularization by Percutaneous Coronary Intervention (PCI) or bypass surgery so; it is important to identify viable myocardium in those patients to improve their long-term survival $[\mathbf{8 , 9}]$

However, revascularization procedures are associated with a higher risk for peri-operative complications and mortality in the same patient so, the distinction of viable and non-viable myocardium is important to select the patients who will benefit most from interventional or surgical revasculariza- 
tion and to prevent patients with scarred myocardium from risks of revascularization therapy [9].

Non-invasive imaging plays an important role in patients with known or suspected myocardial infarction. It helps to assess myocardial perfusion, viability, thickness, motion, and the effects of fibrosis on the kinetics of radiolabelled and paramagnetic contrast agents. The commonly used imaging techniques are low-dose dobutamine str ess echo, SPECT and cMRI which provides not only morphological, but also functional and metabolic information on the heart $[9,10]$.

Patient prognosis after an acute Myocardial Infarction (MI) is influenced by Left Ventricular (LV) function, infarct size and the status of the microvasculature within the infarct zone. As a result, patient risk after an acute MI can be identified by multi-modality imaging approach with echocardiography, ventriculography, and/or Cardiacmagnetic Resonance (CMR) imaging.CMR has the unique ability to assess global and regional LV function post-MI, quantify infarct size with Late Gadolinium Enhancement (LGE), assess the status of the microvasculature by identifying Microvascular Obstruction (MO), and evaluate the area at risk with T2-weighted imaging of myocardial edema. Thus, CMR is uniquely positioned to evaluate the post-infarct patient [11].

In chronic MI, evaluation of the transmurality of infarction relates to the likelihood of functional recovery of dysfunctional myocardium. LGE plays an important role in the assessment of patients with congestive heart failure and chronic ischemic heart disease before revascularization [11]

In clinical practice, various cMRI techniques are used to detect myocardial viability and accordingly predict regional wall motion improvement after revascularization therapy. They include; cine imaging for regional and global systolic function, T2-weighted imaging with fat suppression for myocardial edema and area at risk, first-pass perfusion imaging for microvascular occlusion (rest) and ischemia (rest-stress), late gadolinium enhanced imaging for detection and quantification of myocardial scarring and fibrosis $[\mathbf{9 , 1 2}]$.

The aim of this study was to assess the role of cMRI as a non-invasive tool in the evaluation of myocardial viability in patients with ischemic heart disease.

Myocardial function can be assessed by cMRI and echocardiography. The latter is the most widely used technique due to its easy performance as a bedside rapid screening tool, no radiation exposure and its cheap cost. However, sometimes there are difficulties in defining endocardial contours in patients with limited image quality and poor echo imaging window especially in patients with advanced pulmonary disease, high body mass index, and those who underwent thoracic surgery. So, echo cannot evaluate SWMA in all patients $[12,13]$

Cardiac MRI is now considered the gold standard modality for the assessment of ventricular function and volumes [11]. One of the major strengths of cMRI is the accurate assessment of ventricular function as the excellent visualization of the myocardium and its endo-and epicardial borders on cineRI allows monitoring of changes in wall thickening and wall motion throughout the cardiac cycle and so, LV volumes and EF obtained by cMRI are highly accurate, reproducible and well validated $[\mathbf{1 4 , 1 5}]$

In the current study, myocardial contractility and function was assessed by both echocardiography and cMRI and results were compared. Good agreement between echocardiography and MRI for the estimation of Ejection Fraction (EF) was noted. The mean EF by cMRI was $43.92 \%$ and the mean EF by echocardiography was $39.87 \%$ with mean difference was $4.05 \%$. These findings agreed with Hoffmann et al., 2005 [16] who stated that the mean differences between EF defined by echocardiography images and EF by cMRI were below $5 \%$.

Gardner et al., 2009 [17] found that LVEF correlated moderately well between MRI and echocardiography. However, echo underestimated LVEF compared to cMRI by $4 \%$ points. This also agrees with our study.

One of the important predictors of myocardial viability is regional contractility at rest as the aim of different treatment strategies is to preserve the contractile function of the heart [18]

In the current study, SWMA was assessed by echocardiography for prediction of scar tissue. It was found that 56 territories showed SWMA; 36 $(48 \%)$ of them showed scar by LGE and 20 territories $(26.6 \%)$ showed no scar (i.e $26.6 \%$ of territories are only dysfunctional and completely viable). 19 territories (25.4\%) showed no SWMA with 5 of them showed scar by cMRI. So, sensitivity of echo for the detection of SWMA was $87.8 \%$, its specificity $=41.2, \mathrm{PPV}=64.3 \%, \mathrm{NPV}=73.7 \%$ and accuracy $=66 \%$.

As regard the detection of SMWA by cMRI, it was found that 58 territories ( $77 \%$ of total number 
of 75 territories) showed SWMA; where 40 of them $(53 \%)$ showed scar by LGE and 18 territories (24\%) showed no scar (i.e $24 \%$ of the territories are only dysfunctional and completely viable). 17 territories (23\%) showed no SWMA where only 3 of them (4\%) showed scar by cMRI. So, sensitivity of cMRI for the detection of SWMA was $93.6 \%$, its specificity $=44.4 \%, \mathrm{PPV}=67.82 \%, \mathrm{NPV}=91.67 \%$ and accurac $=72 \%$.

Two cMRI techniques are currently used to assess myocardial viability: Late gadolinium enhancement (LGE) CMR, a technique unique to cMRI that defines the trans-mural extension of scar, and dobutamine stress CMR (DSMR), a method analogous to dobutamine echocardiography that measures the contractile reserve of dysfunctional myocardium and is interpreted by visual analysis $[19,20]$

Hedgire et al., [21] found that the superiority of LGE in assessing myocardial viability in acute or chronic myocardial infarction has been well established with sensitivity as high as 99 or $94 \%$, respectively. The extent of LGE of the transmural thickness to predict functional recovery post revascularization is widely validated and has become a standard clinical practice. LGE images can also identify thrombi in the LV, which can cause catastrophic outcomes as a result of systemic embolization. It can also identify MVO as a persistent hypo-intense (dark) area, surrounded by abnormal late gadolinium on delayed images.

In the current study, 2 segments showed 1-25\% transmural extent of the myocardial wall infarction, 34 segments showed $26-50 \%$ transmural extent of infarction, 8 segments showed $51-70 \%$ transmural extent of infarction and 120 segments showed 71$100 \%$ transmural extent of infarction while 261 segments showed no enhancing scar tissue however, no follow-up studies were performed for those patients to evaluate functional improvement after revascularization.

Casolo et al., [22] evaluated the diagnostic capabilities of the different imaging modalities in detecting CAD. LGE showed the best diagnostic capabilities (sensitivity $98 \%$, specificity $84 \%$ ) and did not improve by evaluating its combination with wall motion abnormality which showed sensitivity of $80 \%$, specificity of $84 \%$, PPV of $92 \%$, NPV of $67 \%$.

Souto et al., [7] have divided the groups of delayed enhancement into five, according to the probability of contractile recovery of the studied segment. The first group included patients with no evidence of enhancing scar (i.e., zero fibrosis/ infarction), it showed a high probability (around $80 \%$ ) of contractile improvement.

The second included patients with $1-25 \%$ transmural hyper-enhancement, the probability of improvement decreased to $60 \%$. The third group included patients with $26-50 \%$ transmural hyperenhancement, the probability of contractility improvement was around $40 \%$. The fourth group included patients showing 51-75\% transmural hyper-enhancement, the contractility improvement was only about $10 \%$. In this group, the decision between revascularization and clinical treatment had to be widely discussed as the risks of angioplasty or surgery may outweigh the benefits of revascularization. The fifth group comprises those having more than $75 \%$ of the area of the myocardial segment hyper-enhanced, the potential of contractile recovery is less than $1 \%$.

In this study, we tried to evaluate other benefits of CMR over echocardiography in the detection of complications associated with ischemic cardiomyopathy such as thinning of myocardial walls, LV thrombus, valvular lesions and micro-vascular occlusion.

Bax et al., [23] found that areas with contractile dysfunction and markedly thinned wall $(<6 \mathrm{~mm}$ end-diastolic wall thickness) have a low likelihood of viability and functional recovery. However, dysfunctional regions with preserved wall thickness do not always improve in function, and this is related to the presence of sub-endocardial scar (and the remainder of the wall being normal). They found that sensitivity of EDWT for the prediction of myocardial viability was $95 \%$ with specificity of $41 \%$, PPV of $56 \%$ and NPV of $92 \%$.

In the current study, thinning of myocardial walls in the chronic state was detected by cMRI in 13 patients of the 25 patients (52\%), only 1 patient (4\%) showed LV wall thinning by both echocardiography and cMRI while the other 12 patients were assessed to have normal myocardial thickness by echocardiography.

In the current study, 3 patients (12\%) were proven to have LV thrombus by cMRI Fig. (1), two of them (8\%) had LV thrombus on MRI which was not appreciated by echocardiography and 1 patient (4\%) was suspected to have LV thrombus by echocardiography, revealed to be free.

Also cMRI was valuable in the diagnosis of valvular lesions as 7 patients (28\%) were diagnosed to have valvular lesions by cMRI Figs. $(3,4)$; only 
4 of them (16\%) showed valvular lesions by both echocardiography and cMRI and 3 patients $(12 \%)$ were diagnosed to have valvular lesions by cMRI while they were assessed to have no valvular lesions by echocardiography.

Despite prompt recanalization of the Infarct Related Artery (IRA), perfusion of the microcirculatory bed does not always occur. Histo-pathological studies have demonstrated that at the infarct core (endocardial), necrosis occurs rapidly with myocardial and capillary endothelial cells perishing simultaneously. Capillaries can become obstructed by cellular debris, resulting in non-perfusion of the infarct core, despite IRA recanalization. This is known as MVO and can be indicated at angiography, as "no reflow" [11]

When it is found, it is exclusively associated with non-viability; thus, the detection of transmural enhancement with a mid-wall hypo-intense zone denotes severe myocardial injury and it is associated with worse prognosis after acute myocardial infarction due to its negative effects on LV function, wall thickness and stiffness, and remodelling, and subsequent risk of heart failure and arrhythmias $[\mathbf{7 , 1 1 , 1 3 ]}$.

In the current study, 9 patients of 25 patients (3 6\%) had MVO appreciated on early gadolinium enhancement images as a zone of non-enhancement Fig. (2) denoting the presence of acute myocardial infarction on top of ischemic heart disease however, no follow-up studies were performed for those patients to evaluate functional improvement after revascularization.

Morphological black blood T2-weighted MRI can detect myocardial edema in patients with acute MI. Because both acute and chronic MI demonstrate late gadolinium enhancement, T2weighted MRI is useful to distinguish the acute infarction from chronic one. In acute MI, the myocardium that exhibits edema, but no late gadolinium enhancement, corresponds to the "area at risk". This can be used to guide targeted therapy at the vulnerable, but potentially salvageable myocardium, surrounding the necrotic and irreversibly damaged region [24]

In the studied cases, it was found that 12 patients (48\%) showed abnormally high signal intensity in T2WI in the jeopardized myocardium denoting myocardial edema and hence acute myocardial infarction Figs. $(1,2)$ and by its correlation with extension of the scar tissue in LGE, it was possible to determine the area at risk i.e salvageable myocardium.
This study had a number of limitations. First of all, the study included a relatively small number of population to evaluate myocardial viability in patients with ischemic heart disease due to relatively short duration of the study and decreased referral of patients. Second, we were not able to perform dobutamine stress cMRI due to the risk of administering dobutamine infusion to a patient in the magnet as positive inotropic stimulation in patients with CAD is associated with a wellrecognized risk of eliciting arrhythmic or ischemic events and the position of the patient within the magnet impairs physician-patient interaction. In addition, the diagnostic utility of ECG monitoring is diminished within the magnetic field. So, as previously mentioned combination between LGE and SWMA by MRI can give an idea (although this can't be highly accurate) about contractile reserve. Another limitation was that no follow-up studies were performed for those patients to evaluate functional improvement after revascularization.

\section{Conclusion:}

Cardiac Magnetic Resonance (cMRI) has proven to be a well-established method to assess myocardial viability through a series of different techniques. In cMRI examination, we were able to evaluate the following in one setting; assessment of both RV and LV function, volumetric assessment of RV and LV, differentiate acute from chronic cases, assessment of both infarct size and transmurality, assessment of RV involvement in the infarct, assessment of microvascular obstruction, detection of intracavitary thrombus and detection of valvular lesions.

\section{Competing interests:}

The authors declare that they have no competing interests.

\section{References}

1- ALLMAN K.C., SHAW L.J., HACHAMOVITCH R., et al.: Myocardial viability testing and impact of revascularization on prognosis in patients with coronary artery disease and left ventricular dysfunction: A meta-analysis. J. Am. Coll. Cardiol.; 39: 1151-8, 2002.

2- KAANDORP T.A., LAMB H.J. and WALL E.E.: Cardiovascular MRI to assess myocardial viability in chronic ischaemic left ventricular dysfunction. Heart; 91: 1359$65,2005$.

3- SCHINKEL A.F., BAX J.J., POLDERMANS D., et al.: Hibernating myocardium: Diagnosis and patient outcomes. Curr. Probl. Cardiol.; 32: 375-410, 2007.

4- ABDELRAHMAN S.F., ALI M.T., SALEM M.A., et al.: Utility of cardiac MRI in the assessment of myocardial viability: Evaluating its role using 3-T machine in correlation with SPECT. The Egyptian Journal of Radiology and Nuclear Medicine; 47: 73-81, 2016. 
5- WAGNER A., MAHRHOLDT H., HOLLY T.A., et al.: Contrast-enhanced MRI and routine Single Photon Emission Computed Tomography (SPECT) perfusion imaging for detection of subendocardial myocardial infarcts: An imaging study. Lancet; 361: 374-9, 2003.

6- SCHVARTZMAN P.R., SRICHAI M.B., GRIMM R.A., et al.: Non-stress delayed-enhancement magnetic resonance imaging of the myocardium predicts improvement of function after revascularization for chronic ischemic heart disease with left ventricular dysfunction. Am. Heart J.; 146: 535-41, 2003.

7- SOUTO A.L.M., SOUTO R.M., TEIXEIRA I.C., et al.: Myocardial Viability on Cardiac Magnetic Resonance. Arq. Bras. Cardiol.; 108: 458-69, 2017.

8- TRAVIN M., and BERGMANN S.R.: Assessment of Myocardial Viability. Semin. Nucl. Med.; 35: 2-16, 2005.

9- JORN J.W.: Assessment of myocardial viability by MR imaging. Journal of Eur. Radiol.; 13: 52-61, 2003.

10- THYGESEN K., ALPERT J.S., and WHITE H.D.: Universal Definition of Myocardial Infarction. Journal of the American College of Cardiology; 50: 2173-95, 2007.

11-KHAN J.N. and MCCANN G.P.: Cardiovascular magnetic resonance imaging assessment of outcomes in acute myocardial infarction. World Journal of Cardiology; 9: 109-33, 2017.

12-APPELBAUM E.A., PETERS D.C., NEZAFAT R., et al.: Left ventricular infarct size, peri-infarct zone, and papillary scar measurements: A comparison of high-resolution 3D and conventional 2D late gadolinium enhancement cardiac MRI. J. Magn. Reson. Imaging; 30: 794-800, 2009.

13- DEWEY M., MULLER M., EDDICKS S., et al.: Evaluation of global and regional left ventricular function with 16-slice computed tomography, biplane cineventriculography, and two-dimensional transthoracic echocardiography comparison with magnetic resonance imaging. J.A.C.C.; 48: 2034-44, 2006.

14- KÜHL H.P., SPUENTRUP E., WALL A., et al.: Assessment of myocardial function with interactive non-breathhold real-time MR imaging: Comparison with echocardiography and breath-hold cine MR imaging. Radiology; 231: 198-207, 2004.

15- PENNELL D.J., MOON J.C., FISHER N.G., et al.: Detection of apical hypertrophic cardiomyopathy by cardi- ovascular magnetic resonance in patients with nondiagnostic echocardiography. Heart, 90: 645-9, 2004.

16- HOFFMANN R., VON BARDELEBEN S., CATE F.T., et al.: Assessment of systolic left ventricular function: A multi-centre comparison of cine ventriculography, cardiac magnetic resonance imaging, unenhanced and contrastenhanced echocardiography. European Heart Journal; 2: 607-16, 2005.

17- GARDNER B.I., BINGHAM S.E., ALLEN M.R., et al.: Cardiac magnetic resonance versus transthoracic echocardiography for the assessment of cardiac volumes and regional function after myocardial infarction: An intrasubject comparison using simultaneous intra-subject recordings. Cardiovasc Ultrasound; 7: 38, 2009.

18- GERBER B.L., ROCHITTE C.E., BLUEMKE D.A., et al.: Relation between Gd-DTPA contrast enhancement and regional inotropic response in the periphery and center of myocardial infarction. Circulation; 104: 998-1004, 2001.

19- GROVER S., SRINIVASANA G. and SELVANAYAGAMA J.B.: Evaluation of Myocardial Viability with Cardiac Magnetic Resonance Imaging. Prog. Cardiovasc. Dis.; 54: 204-14, 2011.

20- EMERSON C., GUILHERME V., SARMENTO-LEITE R., et al.: Assessing Myocardial Viability and Infarct Transmurality With Left Ventricular Electromechanical Mapping in Patients With Stable Coronary Artery Disease Validation by Delayed-Enhancement Magnetic Resonance Imaging. Circulation; 6: 957-61, 2002.

21- HEDGIRE S.S., OSBORNE M., VERDINI D.J., et al.: Updates on Stress Imaging Testing and Myocardial Viability With Advanced Imaging Modalities. Curr. Treat. Options Cardio. Med.; 19: 1-12, 2017.

22- CASOLO G., MINNECI S., MANTA R., et al.: Identification of the ischemic etiology of heart failure by cardiovascular magnetic resonance imaging: Diagnostic accuracy of late gadolinium enhancement. American Heart Journal; 151: 101-8, 2006.

23- BAX J.J. and DELGADO V.: Myocardial viability as integral part of the diagnostic and therapeutic approach to ischemic heart failure. Journal of Nuclear Cardiology; 22: $229-45,2015$

24- ISHIDA M., KATO S. and SAKUMA H.: Cardiac MRI in Ischemic Heart Disease. Circulation. J.; 73: 1577-88, 2009.
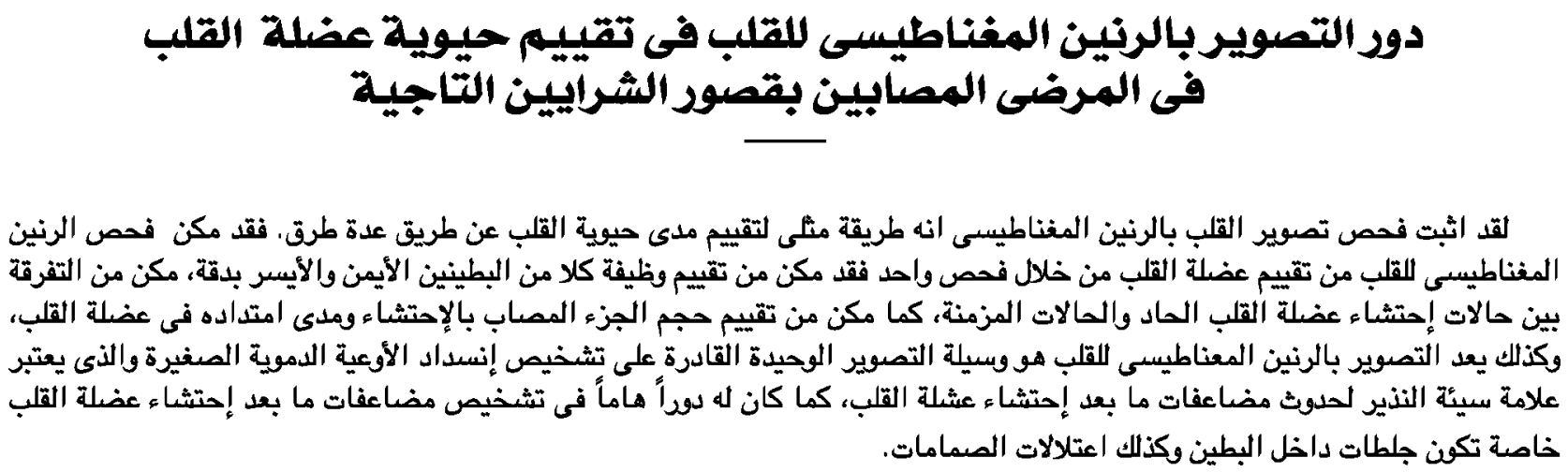